\title{
Phase spectrum estimation of the seismic wavelet based on a criterion function
}

\author{
Yu Yongcai ${ }^{*}$, Wang Shangxu ${ }^{1}$, Yuan Sanyi ${ }^{1}$ and Qi Pengfei ${ }^{2}$ \\ ${ }^{1}$ State Key Laboratory of Petroleum Resources and Prospecting, China University of Petroleum, Beijing 102249, China \\ ${ }^{2}$ School of Geophysics and Geoinformation Technology, China University of Geosciences, Beijing 100083, China
}

(C) China University of Petroleum (Beijing) and Springer-Verlag Berlin Heidelberg 2012

\begin{abstract}
Phase spectrum estimation of the seismic wavelet is an important issue in high-resolution seismic data processing and interpretation. On the basis of two patterns of constant-phase rotation and root transform for wavelet phase spectrum variation, we introduce six sparse criteria, including Lu's improved kurtosis criterion, the parsimony criterion, exponential transform criterion, Sech criterion, Cauchy criterion, and the modified Cauchy criterion, to phase spectrum estimation of the seismic wavelet, obtaining an equivalent effect to the kurtosis criterion. Through numerical experiments, we find that when the reflectivity is not a sparse sequence, the estimated phase spectrum of the seismic wavelet based on the criterion function will deviate from the true value. In order to eliminate the influence of non-sparse reflectivity series in a single trace, we apply the method to the multi-trace seismogram, improving the accuracy of seismic wavelet phase spectrum estimation.
\end{abstract}

Key words: Seismic wavelet, phase spectrum, criterion function, sparse

\section{Introduction}

Seismic wavelet estimation is a key issue in seismic data processing and interpretation. In particular, the phase spectrum estimation is paramount. It directly impacts on the results of forward modeling, deconvolution and inversion (Lu, 2005; Lu et al, 2007; Yuan et al, 2009; Yu et al, 2011). In real data, deterministic methods are often used to estimate the seismic wavelet by utilizing well logs. However, well logs are not always available, and often different well logs predict different results of wavelet estimation. The difference is mainly manifested in the phase spectrum (Edgar and van der Baan, 2011). Thus it is required for statistical approach to estimate the phase spectrum of the wavelet from the seismic data alone. Levy and Oldenburg (1987), Longbottom et al (1988), and White (1988) proposed a method for wavelet phase spectrum estimation. On the assumption that the wavelet phase is a frequency-independent constant, they estimate the wavelet phase spectrum by constant-phase rotation and kurtosis criterion maximization. Longbottom et al (1988) tested some marine data and found that wavelets derived in seismic-to-well ties often have a near-constant phase, which justifies that constant-phase rotation in wavelet phase spectrum estimation is feasible. However, when the wavelet phase spectrum varies with frequency, constant-phase rotation is inappropriate and we need to change the wavelet phase spectrum by root transform.

*Corresponding author. email: yuyongcaiahcai@126.com Received July 29, 2011
Kurtosis belongs to sparse criterion, which was first applied to blind deconvolution by Wiggins (1978). Many geophysicists have studied this criterion and proposed many improved criteria and other sparse criteria, such as Lu's improved kurtosis criterion, the parsimony criterion, exponential transform criterion, Sech criterion, Cauchy criterion, and the modified Cauchy criterion. These criteria all obtain satisfying effects in deconvolution or inversion.

In this paper, we first discuss the two patterns of wavelet phase spectrum variation. Then, similarly to the wavelet phase spectrum estimation based on the kurtosis criterion, we combine Lu's improved kurtosis criterion, the parsimony criterion, exponential transform criterion, Sech criterion, Cauchy criterion, and the modified Cauchy criterion with the two patterns of wavelet phase variation respectively, to estimate the seismic wavelet phase spectrum, and discuss the influence of non-sparse reflectivity series to the method. Finally, we apply the method to multi-trace seismograms to improve the accuracy of seismic wavelet phase spectrum estimation.

\section{Wavelet phase variation}

\subsection{Constant-phase rotation}

In the conditions when the wavelet phase spectrum is a frequency-independent constant, we can change wavelet phase spectrum by constant-phase rotation. Constant-phase rotation can be implemented either in the frequency domain or in the time domain. Let $W(f)$ be the spectrum of wavelet $w(t), \phi$ be the phase rotation angle, and $w_{\text {rot }}(t)$ be the wavelet 
after phase rotation, whose spectrum is $W_{\text {rot }}(f)$. Then in the frequency domain,

$$
W_{\text {rot }}(f)=W(f) \exp (i \phi)
$$

where $i=\sqrt{-1}$ denotes the imaginary unit. Conducting the inverse Fourier transform of Eq. (1), we can obtain $w_{\text {rot }}(t)$.

In the time domain, we can accomplish constant-phase rotation through Eq. (2) (Levy and Oldenburg, 1987),

$$
w_{\text {rot }}(t)=w(t) \cos \phi+H[w(t)] \sin \phi
$$

where $H[\cdot]$ denotes the Hilbert transform.

Comparing the two implementations, without forward and inverse Fourier transform, the time-domain implementation is faster than the frequency-domain (Arons and Yennie, 1950). In addition, each individual time sample can be treated independently and corresponds to a phase rotation angle, therefore Eq. (2) is conducive to time-varying phase rotations (Van der Baan, 2008).

\subsection{Root transform}

When the wavelet phase spectrum is a frequency-related variable, we can change the wavelet phase spectrum by root transform. Suppose the discrete form of wavelet $w_{\text {rot }}(t)$ is $\left(w_{0}, w_{1}, \ldots, w_{n}\right)$, according to $Z$ transform,

$$
\begin{aligned}
w(t) & =Z^{-1}\left[w_{n}\left(z-\alpha_{1}\right)\left(z-\alpha_{2}\right) \cdots\left(z-\alpha_{n}\right)\right] \\
& =w_{n}\left(-\alpha_{1}, 1\right) *\left(-\alpha_{2}, 1\right) * \cdots *\left(-\alpha_{n}, 1\right)
\end{aligned}
$$

where $Z^{-1}[\cdot]$ denotes inverse $Z$ transform, $\left(\alpha_{1}, \alpha_{2}, \ldots, \alpha_{n}\right)$ denotes the $Z$ transform roots of the wavelet, $*$ denotes convolution. Substituting $\left|\overline{\alpha_{i}}\right|\left(-\frac{1}{\bar{\alpha}}, 1\right)$ for $\left(-\alpha_{i}, 1\right)$ in Eq. (3), which is equivalent to making a symmetry transform on the

unit circle about the $Z$ transform roots of the wavelet. The wavelet amplitude spectrum does not change, only the phase spectrum changes (Yuan and Wang, 2011). Through different combinations of the root transform, we can obtain a series of wavelets which have the same amplitude spectra and different phase spectra. In general, suppose the number of real roots is $N r$, and the number of conjugate complex roots is $N c$, then the number $M$ of wavelets is

$$
M=C_{N r+N c / 2}^{0}+C_{N r+N c / 2}^{1}+\cdots+C_{N r+N c / 2}^{N r+N c / 2}=2^{N r+N c / 2}
$$

where $C_{n}^{m}=\frac{n !}{m !(n-m) !}$, and ! denotes factorial.

\section{Criterion function}

\subsection{Kurtosis criterion}

The kurtosis criterion (Wiggins, 1978), also known as varimax norm, measures the deviation from Gaussian of signal $x(t)$. The expression is

$$
\operatorname{kurt}(x)=N \frac{\sum x^{4}(t)}{\left[\sum x^{2}(t)\right]^{2}}
$$

where $N$ denotes number of samples. The kurtosis of the Gaussian signal is 3 . The more the value deviates from 3 , the greater the degree of deviation from a Gaussian signal.

\subsection{Lu's improved kurtosis criterion}

The kurtosis criterion can be placed in the general expression (Sacchi et al, 1994),

$$
V(x)=\frac{1}{N F(N)} \sum_{i=1}^{N}[q(i) \cdot F(q(i))]
$$

where $F(\cdot)$ is a monotonically increasing function, and $q(i)$ is normalized amplitude, whose expression is

$$
q(i)=\frac{[x(i)]^{2}}{\sum_{j=1}^{N}[x(j)]^{2} / N}
$$

For the kurtosis criterion, $F(q(i))=q(i)$. Considering that kurtosis is sensitive to large reflectivity, Lu and Liu (2007) and $\mathrm{Lu}$ (2009) proposed an improved kurtosis criterion by letting $F(q(i))=\frac{\ln (\cosh (\alpha q(i)))}{\alpha q(i)}$ in Eq. (6), where $\alpha>0$ is a sparsity control parameter. The smaller the value is, the sparser the reflectivity series.

\subsection{Parsimony criterion}

The parsimony criterion is a constraint criterion in deconvolution proposed by Claerbout (1977), whose expression is

$$
Q=\ln \left(\sum_{i}\left|x_{i}\right|^{n}\right)-\sum_{i}\left(\left|x_{i}\right|^{n} \cdot \ln \left|x_{i}\right|^{n}\right) / \sum_{i}\left|x_{i}\right|^{n}
$$

The criterion can be rewritten in another form

$$
Q=-\sum_{i}\left(\left|x_{i}\right|^{n} / \sum_{i}\left|x_{i}\right|^{n}\right) \ln \left(\left|x_{i}\right|^{n} / \sum_{i}\left|x_{i}\right|^{n}\right)=-\sum_{i} p_{i} \ln p_{i}
$$

where $p_{i}=\left|x_{i}\right|^{n} / \sum_{i}\left|x_{i}\right|^{n}$ has the property of probability distribution, so Eq. (8) can be seen as another form of Shannon entropy. In calculation, the value of $n$ generally takes 3. Compared with the kurtosis criterion, the parsimony criterion has high sensitivity to both strong and weak reflectivity, and can preserve weak reflection information easily.

\subsection{Exponential transform criterion}

Considering the kurtosis criterion is very tolerant of additive noise, Ooe and Ulrych (1979) wished to improve the balance between the noise suppression and the ability to recover small reflection coefficients in minimum entropy deconvolution, and introduced exponential transform to the results of deconvolution 


$$
z_{i}=1-\exp \left\{-x_{i}^{2} / 2 S^{2}\right\}
$$

where $S=\max (x) / C, C$ is a constant. Then obtain the exponential transform criterion

$$
U=\sum_{i=1}^{N} z_{i}^{2} /\left(\sum_{i=1}^{N} z_{i}\right)^{2}
$$

Note that when $C \rightarrow 0$, Eq. (11) becomes kurtosis criterion.

\subsection{Other sparse constraint criteria}

Sacchi (2002) studied Sech and Cauchy criteria in robust statistics, and introduced these criteria to sparse spiking inversion as a regularization factor, obtaining satisfying effects. These criteria have advantages in anti-noise performance and finding strong reflectivity, yet they have some suppression effect on weak reflection information. In order to get a balance between improving seismic data resolution and decreasing the suppression effect on small reflectivity, Zhang et al (2008) used the modified Cauchy criterion, and also obtained good results in sparse spiking inversion. The three criteria are respectively

$$
\begin{aligned}
& J_{\text {Sech }}=\sum_{i=0}^{N} \ln \left(\cosh \frac{x_{i}^{2}}{2}\right) \\
& J_{\text {Cauchy }}=\sum_{i=0}^{N} \ln \left(1+\frac{1}{2}\left(\frac{x_{i}}{\sigma}\right)^{2}\right) \\
& J_{\text {Cauchy_modified }}=\sum_{i=0}^{N}\left(\frac{x_{i}}{\sigma}\right)^{2} /\left(1+\left(\frac{x_{i}}{\sigma}\right)^{2}\right)
\end{aligned}
$$

where $\sigma$ is the damping parameter.

All of above criteria affect deconvolution or inversion. Among them, the kurtosis criterion is the most commonly used. Wiggins (1978) applied kurtosis to deconvolution, and proposed the minimum entropy deconvolution. Based on a simplification of Wiggins's method, Levy and Oldenburg (1987), Longbottom et al (1988), and White (1988) introduced the kurtosis criterion to seismic wavelet phase spectrum estimation, searching the phase spectrum through constantphase rotation and kurtosis maximization. Van der Baan and Fomel (2009) also introduced the kurtosis criterion to nonstationary wavelet phase estimation. In view of the effect of other constraint criteria in deconvolution or inversion, we introduce these criteria to seismic wavelet phase spectrum estimation.

\section{The corresponding criterion function of wavelet phase spectrum variation}

\subsection{Constant-phase wavelet}

When the wavelet has a constant phase, we can change the wavelet phase spectrum by constant-phase rotation. Fig. 1 conducts constant-phase rotation from $-180^{\circ}$ to $180^{\circ}$ for a zero-phase wavelet. We calculate the values of every criterion function about wavelets after constant-phase rotation. Fig. 2 is normalized values of the criteria functions. We can see that the values of these criteria have similar properties. The period of exponential transform criterion is $360^{\circ}$, others are $180^{\circ}$, and the waveforms are similar to sine or cosine functions. In addition, for a zero-phase wavelet, the kurtosis criterion, Lu's improved kurtosis criterion, exponential transform criterion, and Sech criterion are maximum, and the parsimony criterion, Cauchy criterion, and the modified Cauchy criterion are minimum. However, for $\pm 90^{\circ}$ constantphase wavelet, the kurtosis criterion, Lu's improved kurtosis criterion, exponential transform criterion, and Sech criterion are minimum, and the parsimony criterion, Cauchy criterion, and the modified Cauchy criterion are maximum. According to these characteristics, we can pick the phase spectrum of the constant-phase wavelet.

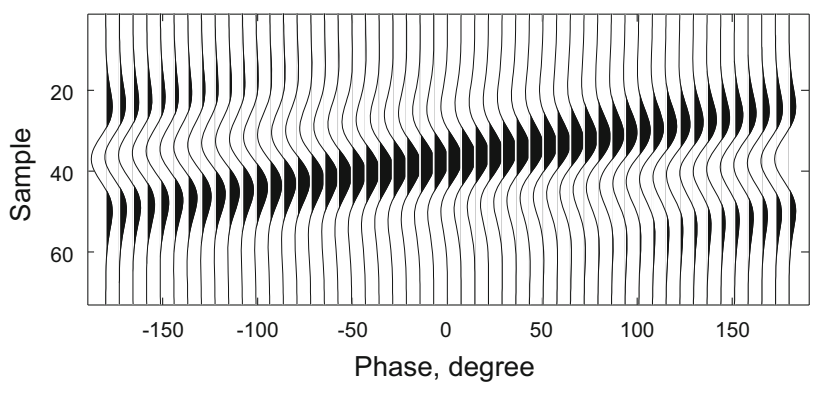

Fig. 1 Wavelets after constant-phase rotation

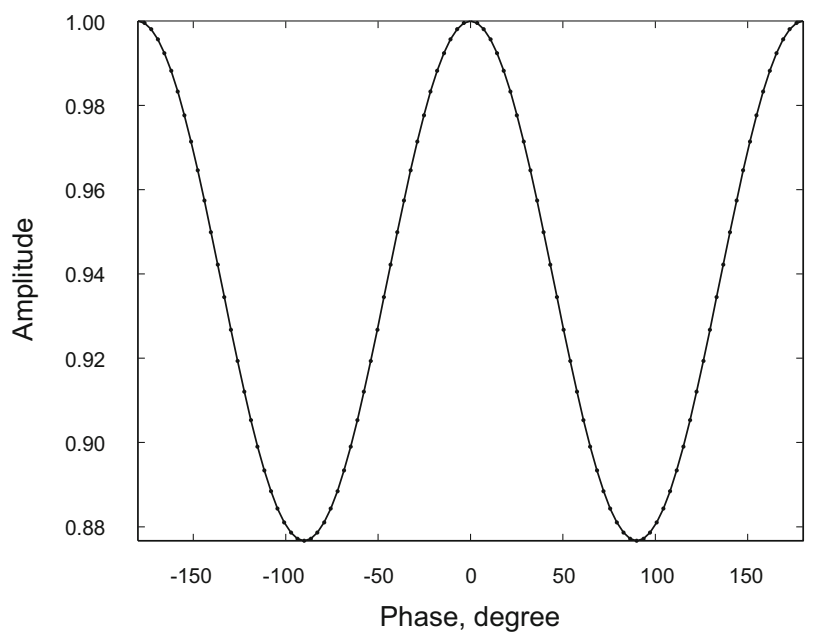

(a)

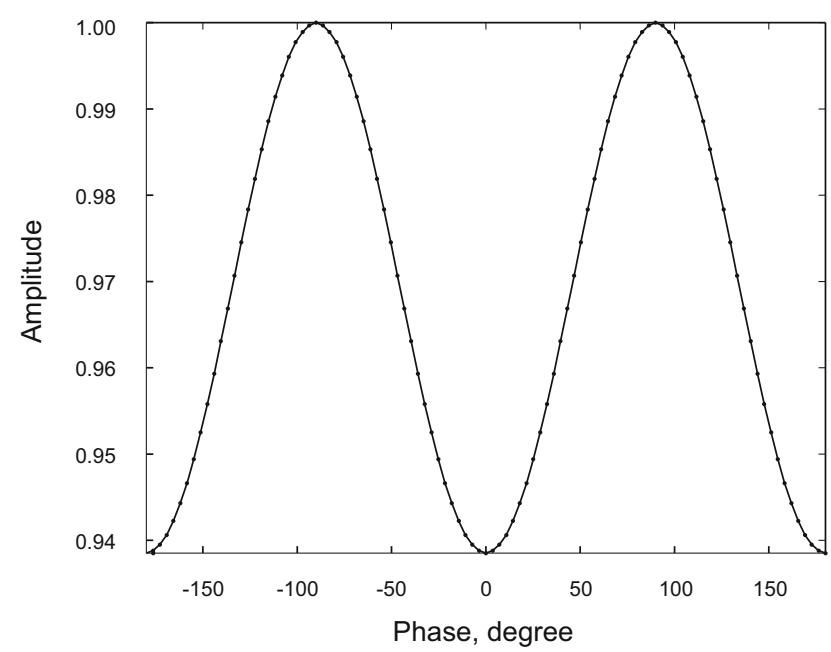

(b) 


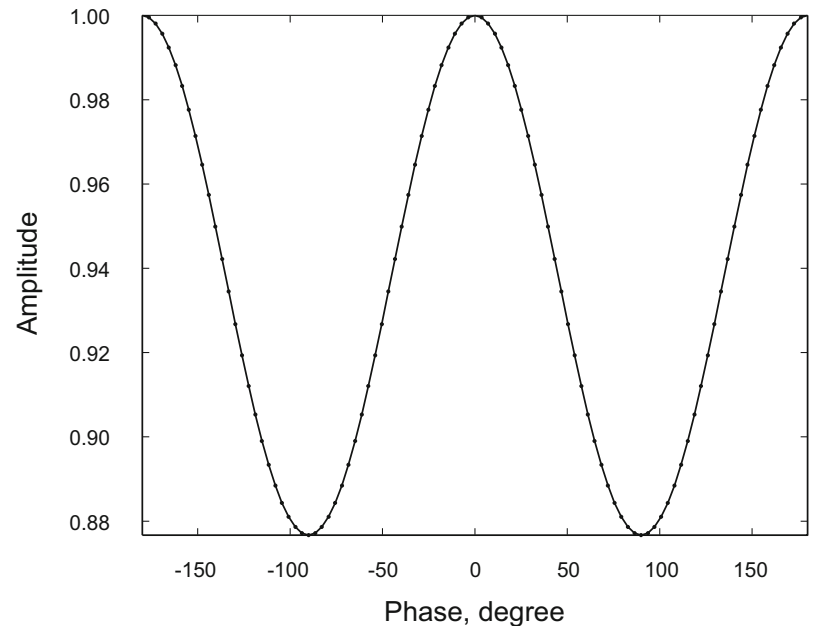

(c)

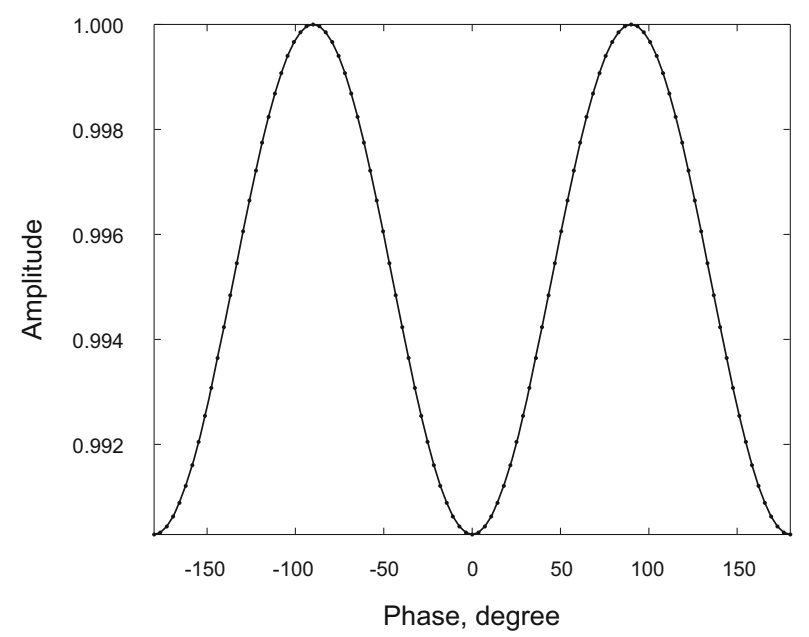

(d)

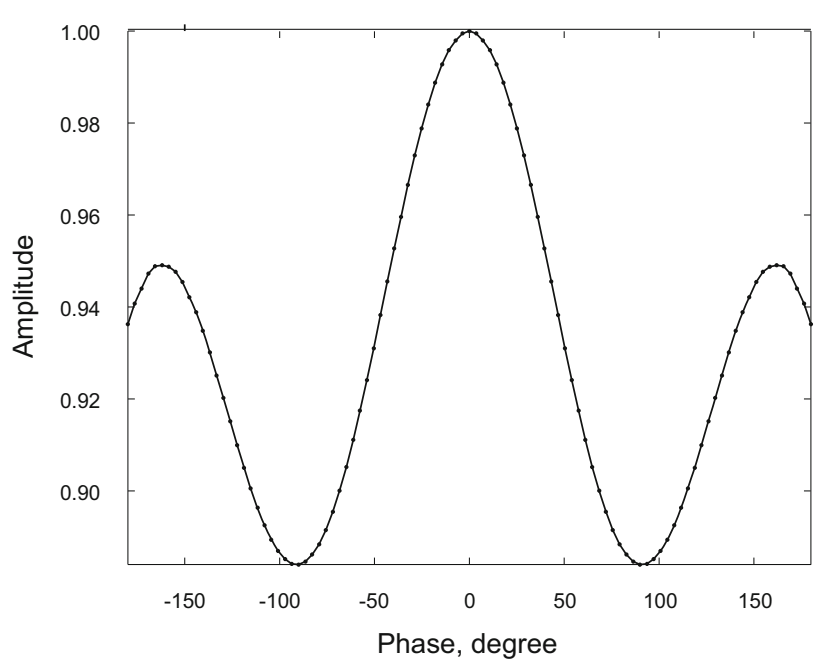

(e)

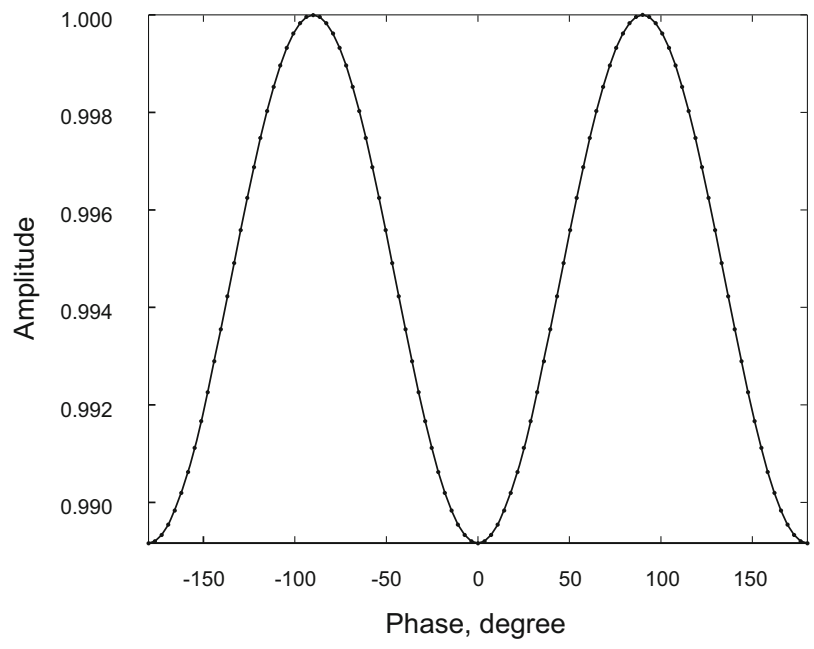

(f)

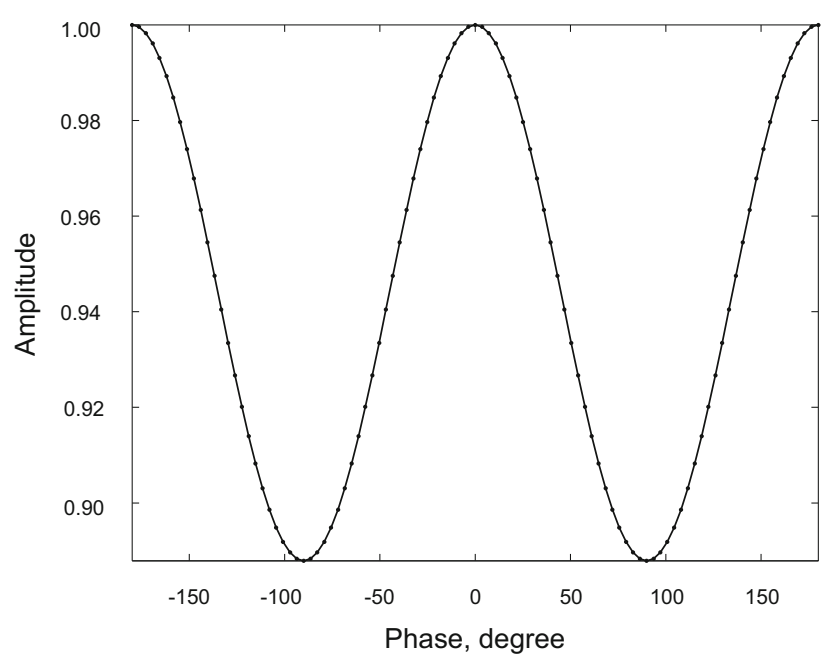

(g)

Fig. 2 The corresponding criteria function of constant-phase wavelets in Fig. 1. (a) kurtosis criterion, (b) parsimony criterion, (c) Lu's improved kurtosis criterion, (d) Cauchy criterion, (e) exponential transform criterion, (f) modified Cauchy criterion, and (g) Sech criterion

\subsection{Non-constant-phase wavelet}

When the wavelet is of non-constant phase, we can change the wavelet phase spectrum by root transform. Given two real roots $1.3798,1.3628$, and four pairs of conjugate complex roots $-0.3338 \pm 1.4296 \mathrm{i},-0.1363 \pm 1.3080 \mathrm{i},-0.7727 \pm 0.9136 \mathrm{i}$, $-1.0489 \pm 0.1858 \mathrm{i}$, we can obtain 64 wavelets (Fig. 3) which have the same amplitude spectra and different phase spectra by root transform. Among the wavelets in Fig. 3, the first is a minimum-phase wavelet, the last is a maximum-phase wavelet, and others are mixed-phase wavelets. Fig. 4 is the criteria function of 64 wavelets in Fig. 3. We can see that the values of these criteria functions have similar properties. The kurtosis criterion, Lu's improved kurtosis criterion, exponential transform criterion, and Sech criterion have the same trend, and the parsimony criterion, Cauchy criterion, 
and the modified Cauchy criterion have the same trend. In addition, the kurtosis criterion, Lu's improved kurtosis criterion, exponential transform criterion, and Sech criterion of $20^{\text {th }}, 24^{\text {th }}, 28^{\text {th }}, 31^{\text {st }}, 34^{\text {th }}, 37^{\text {th }}, 41^{\text {st }}$, and $45^{\text {th }}$ wavelets are maximum, and the parsimony criterion, Cauchy criterion, and the modified Cauchy criterion are minimum. We pick up these 8 wavelets (Fig. 5) and find that their waveforms tend to symmetry. According to these characteristics, we can estimate the phase spectrum of the non-constant-phase wavelet.

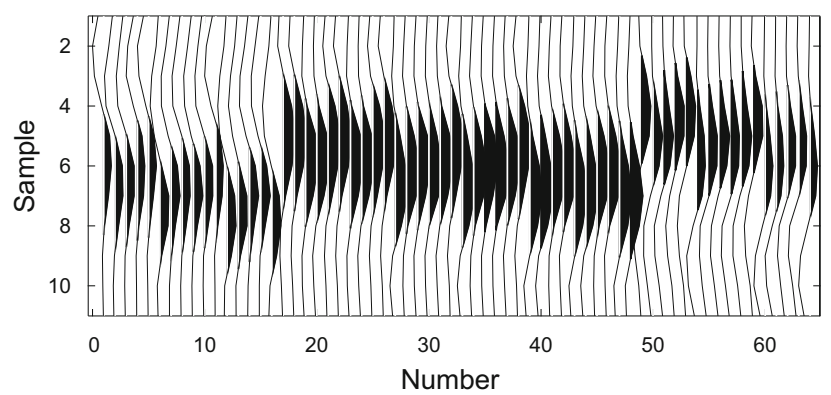

Fig. 3 Mixed-phase wavelets by root transform

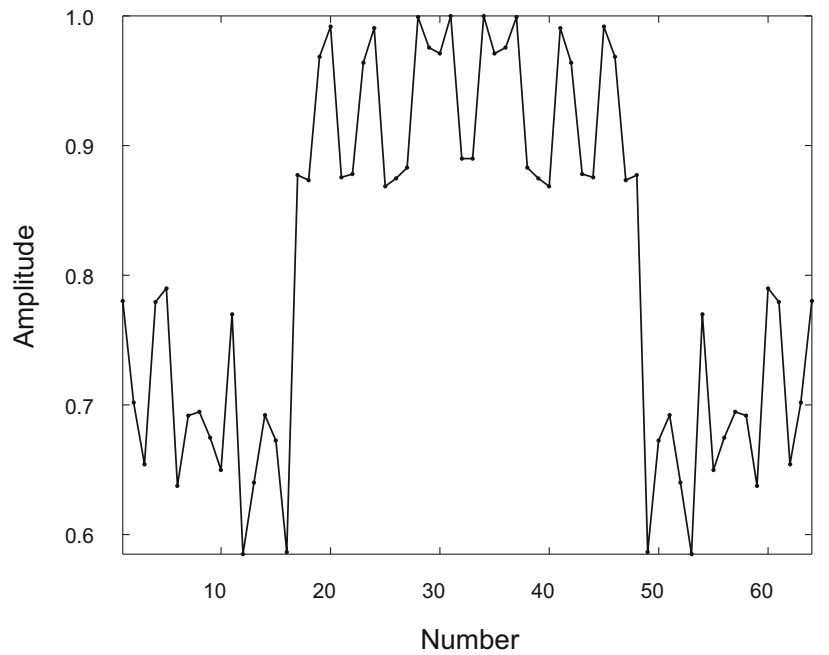

(a)

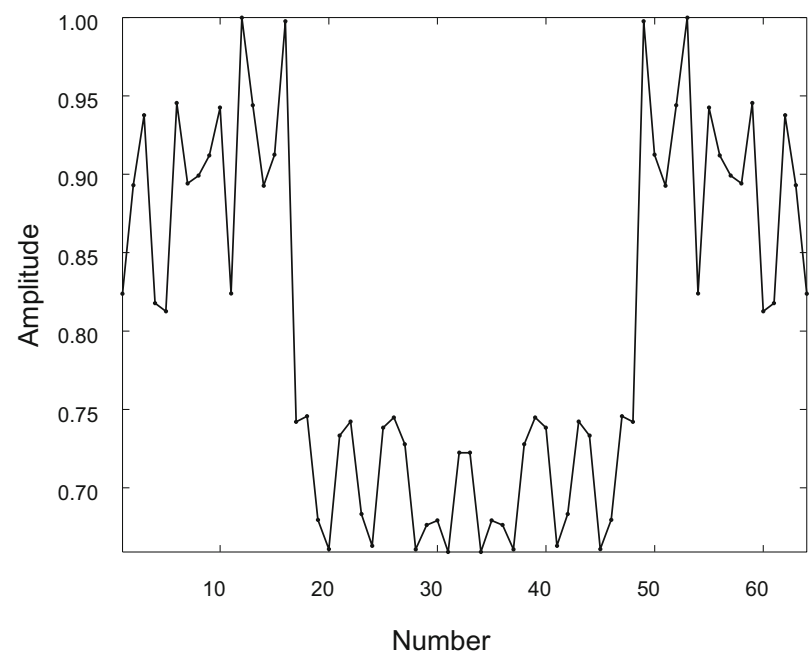

(b)

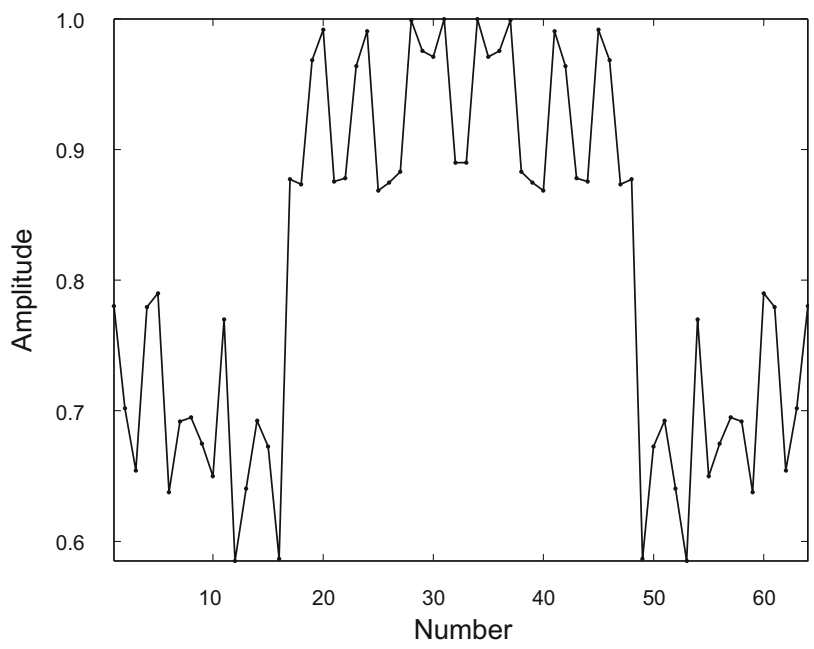

(c)

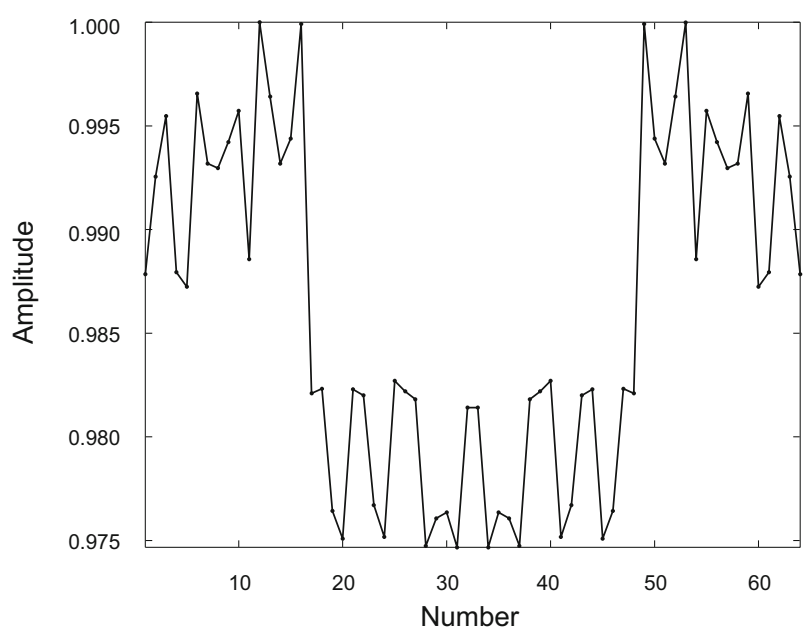

(d)

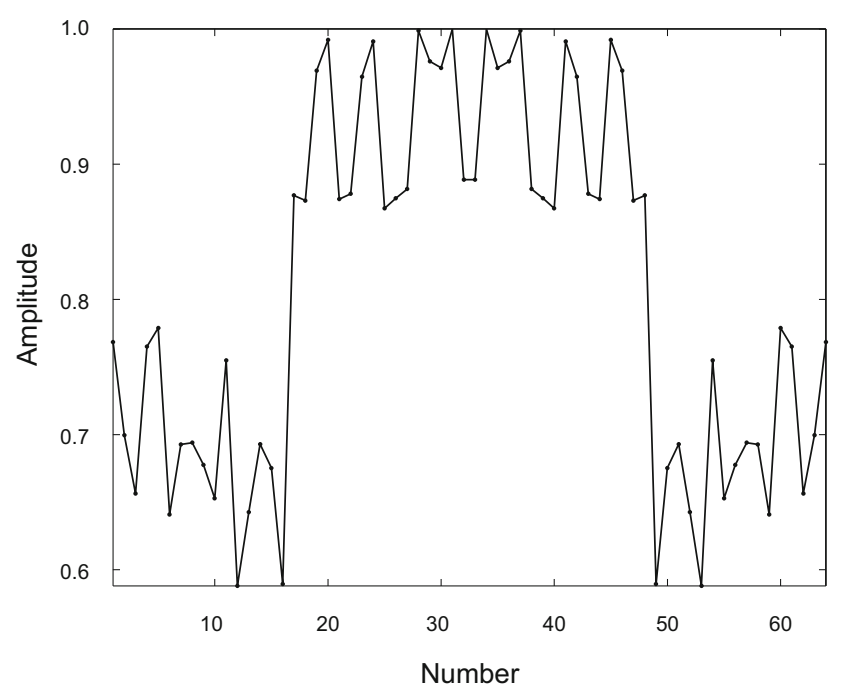

(e) 


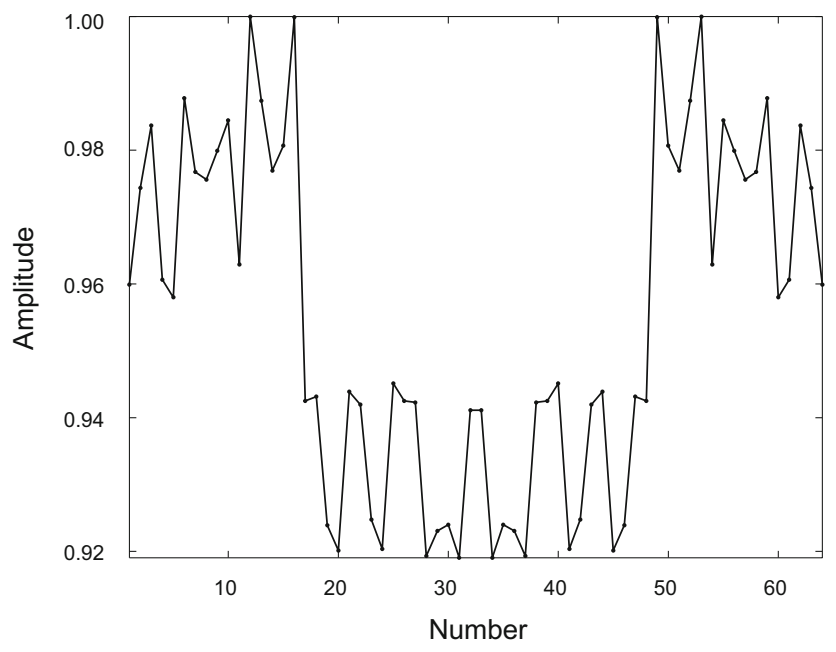

(f)

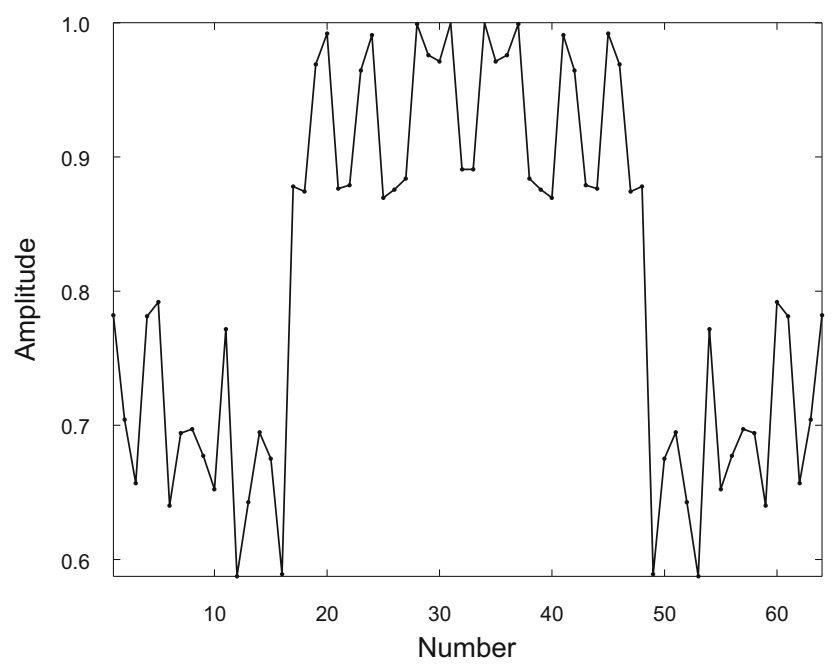

(g)

Fig. 4 The corresponding criteria function of non-constant-phase wavelets in Fig. 3. (a) kurtosis criterion, (b) parsimony criterion, (c) Lu's improved kurtosis criterion, (d) Cauchy criterion, (e) exponential transform criterion, (f) modified Cauchy criterion, and (g) Sech criterion

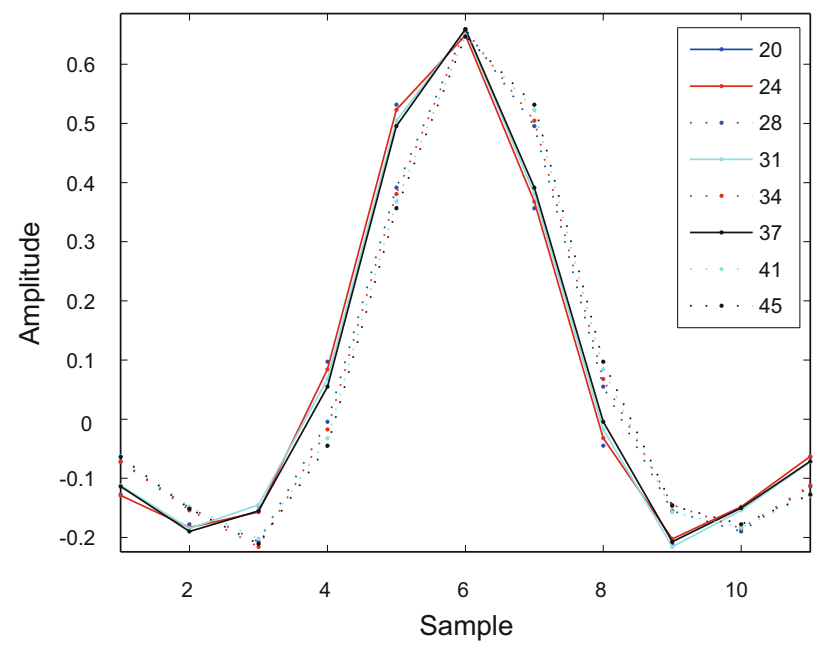

Fig. 5 The $20^{\text {th }}, 24^{\text {th }}, 28^{\text {th }}, 31^{\text {st }}, 34^{\text {th }}, 37^{\text {th }}, 41^{\text {st }}$, and $45^{\text {th }}$ wavelets in Fig. 3

\section{Phase spectrum estimation of the seismic wavelet based on criterion functions}

According to the Robinson convolution model (Robinson, 1967), the seismic record $s(t)$ can be represented by the convolution of the seismic wavelet $w(t)$ and reflectivity series $r(t)$,

$$
s(t)=w(t) * r(t)
$$

For a constant-phase wavelet, we can conduct constantphase rotation on the seismic record from $-90^{\circ}$ to $90^{\circ}$, which is equivalent to making a constant-phase rotation on the wavelet with the same angle, then calculating the criterion function. The phase angle corresponding to the extreme of the criterion function is the phase spectrum of the constant-phase wavelet.

For a non-constant-phase wavelet, we need first to estimate the minimum-phase wavelet from the seismic record by spectral modeling (Rosa and Ulrych, 1991) or the autocorrelation method (Leinbach, 1995), then calculate all the wavelets which have the same amplitude spectra and different phase spectra. Finally subtract every wavelet phase spectrum from the seismic record, and calculate the criterion function. The phase spectrum corresponding to the extreme of criterion function is the phase spectrum of non-constantphase wavelet.

However, in the phase spectrum estimation of the seismic wavelet based on the criterion function, the reflectivity series existed in the calculated values of criterion function. Because the criteria belong to sparse criteria, the sparse feature of the reflectivity series will have an impact on the method.

\subsection{Constant-phase wavelet}

Fig. 6 shows a reflectivity series from real data, which is non-sparse. We convolute it with the constant-phase wavelets in Fig. 1 and obtain the seismic profile (Fig. 7). The profile can be viewed as the result of conducting constant-phase rotation on the seismic record obtained by convolution of the zero-phase wavelet and reflectivity series. We estimate the wavelet phase spectrum using the criteria function, and find that the results all deviate from the true value of $28.8^{\circ}$ (Fig. 8).

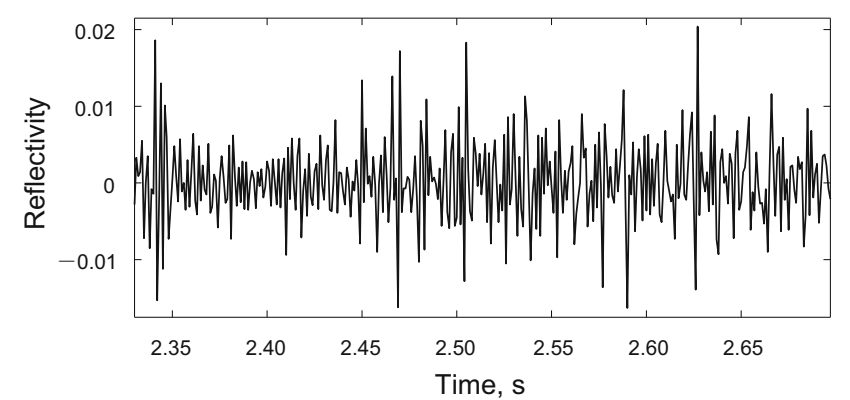

Fig. 6 Reflectivity series from real data 


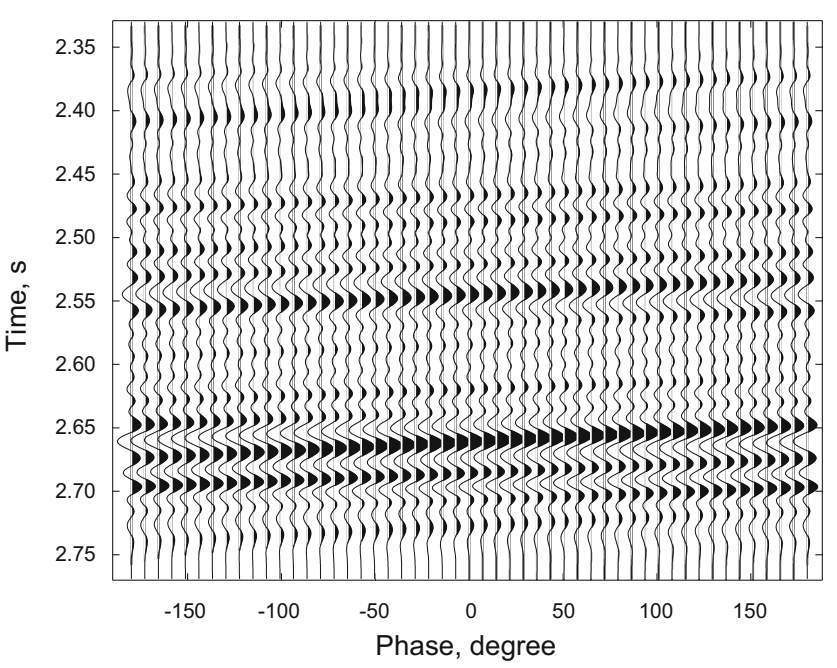

Fig. 7 Seismic traces after constant-phase rotation

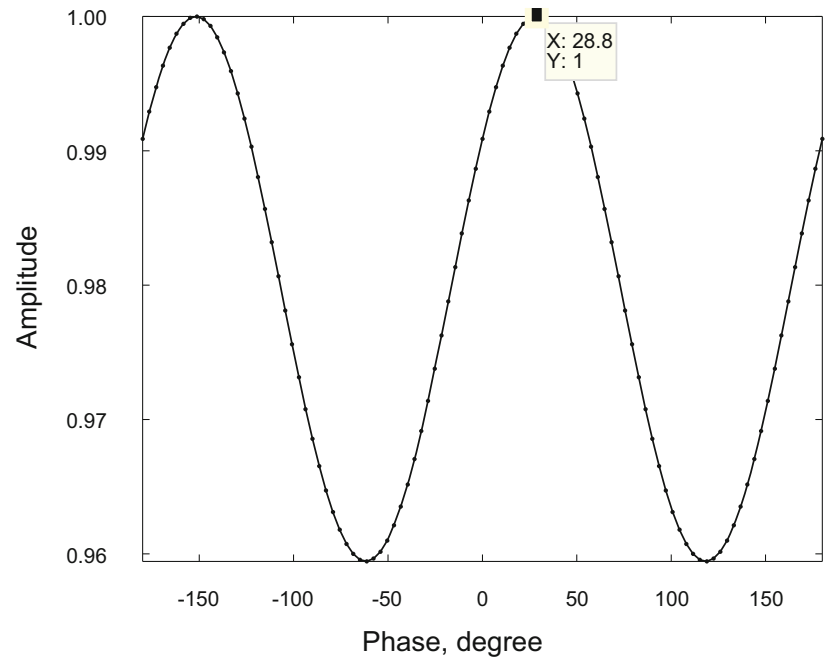

(a)

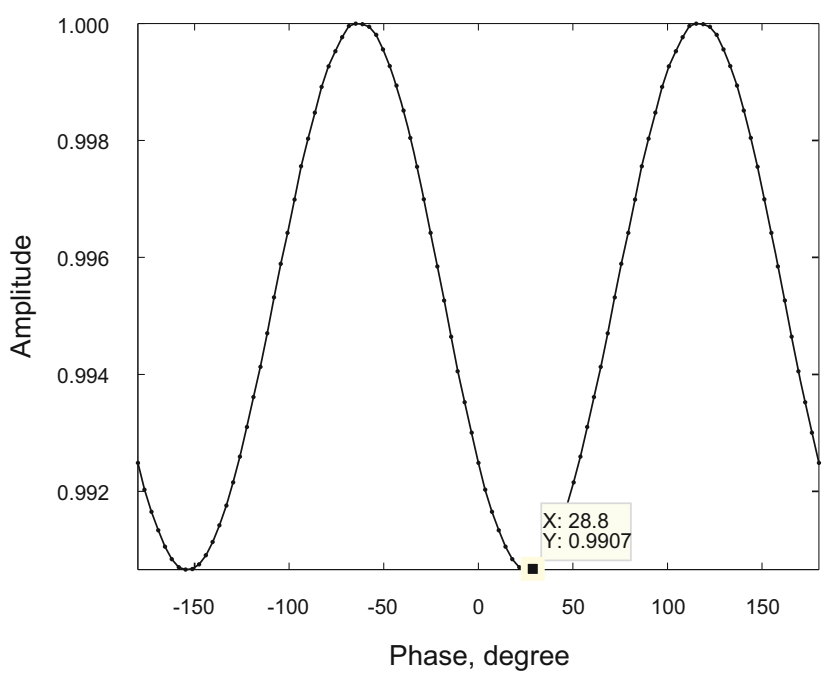

(b)

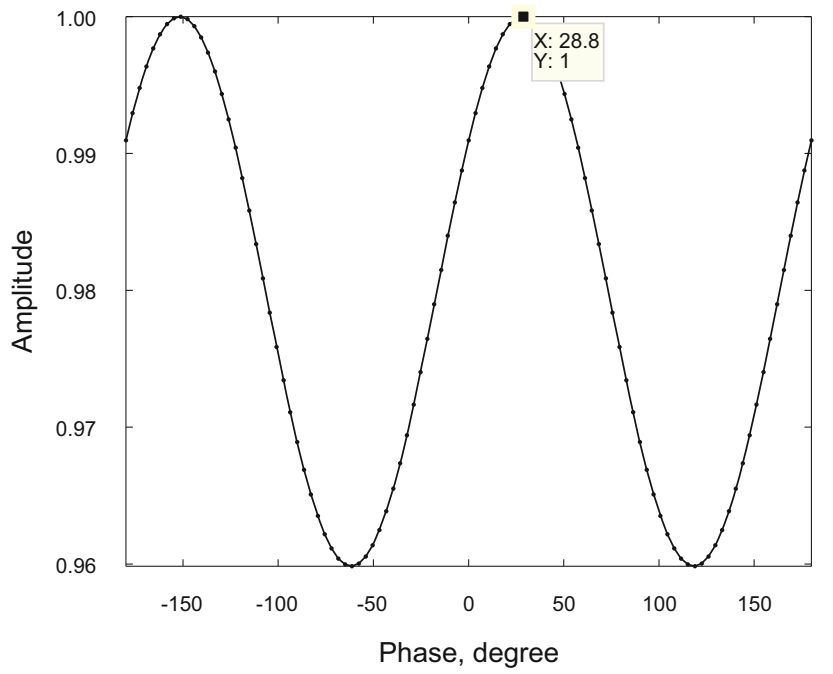

(c)

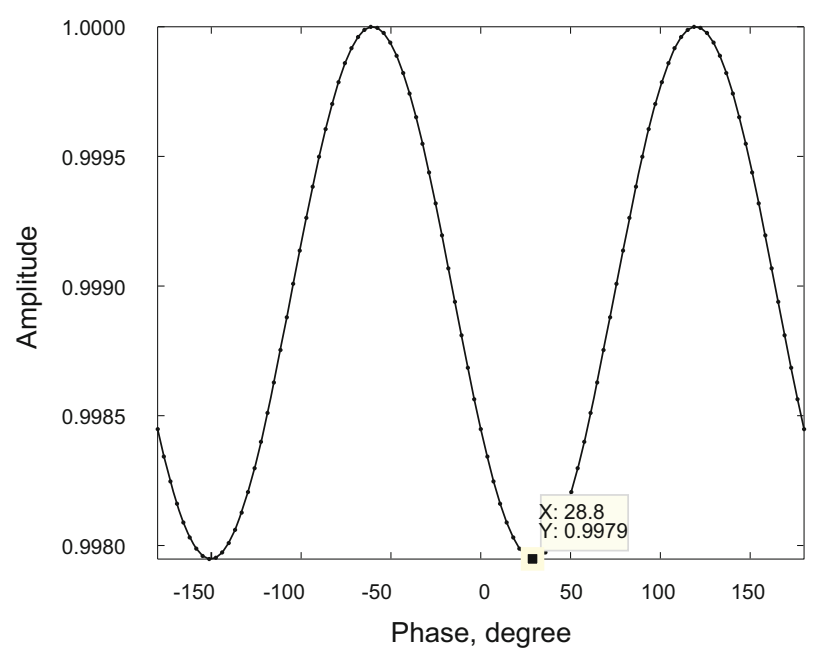

(d)

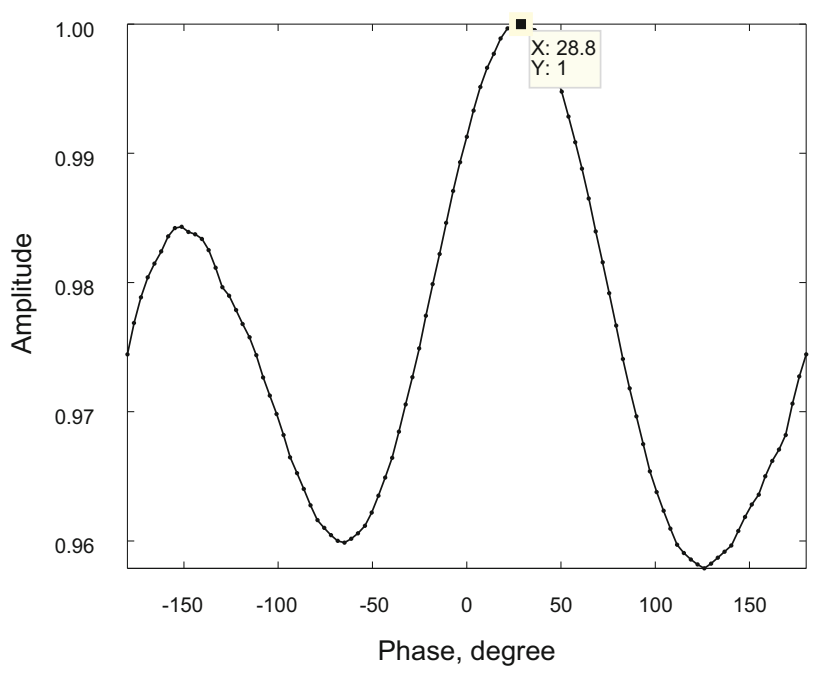

(e) 


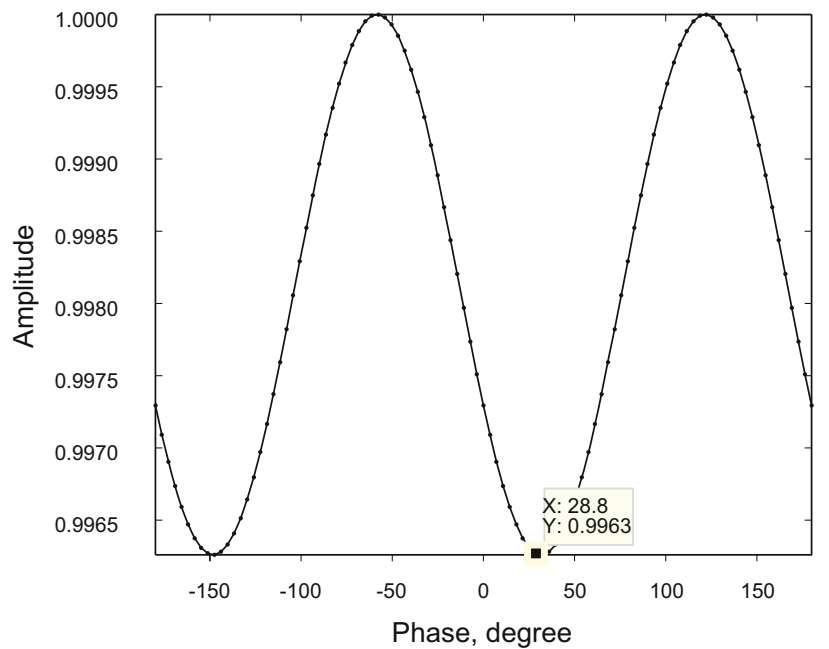

(f)

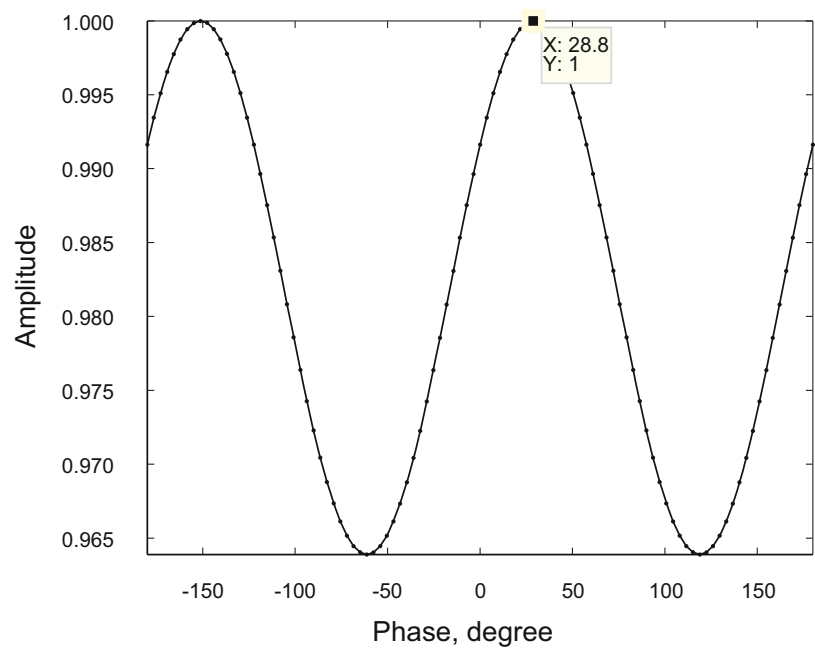

(g)

Fig. 8 The corresponding criteria function of every seismic trace in Fig. 7. (a) kurtosis criterion, (b) parsimony criterion, (c) Lu's improved kurtosis criterion, (d) Cauchy criterion, (e) exponential transform criterion, (f) modified Cauchy criterion, and (g) Sech criterion

\subsection{Non-constant-phase wavelet}

The wavelets in Fig. 3 are convoluted with the reflectivity series in Fig. 6 respectively and the seismic record is obtained (Fig. 9), then we calculate the values of criteria function of every trace (Fig. 10). We can see that the kurtosis criterion, Lu's improved kurtosis criterion, exponential transform criterion, and Sech criterion of $2^{\text {nd }}$ and $8^{\text {th }}$ wavelet are a maximum, and the parsimony criterion, Cauchy criterion, and the modified Cauchy criterion show a minimum. We pick up these wavelets (Fig. 11) and find that their waveforms deviate from symmetry.

Thus we can see that these criteria functions have a similar effect in phase spectrum estimation of the seismic

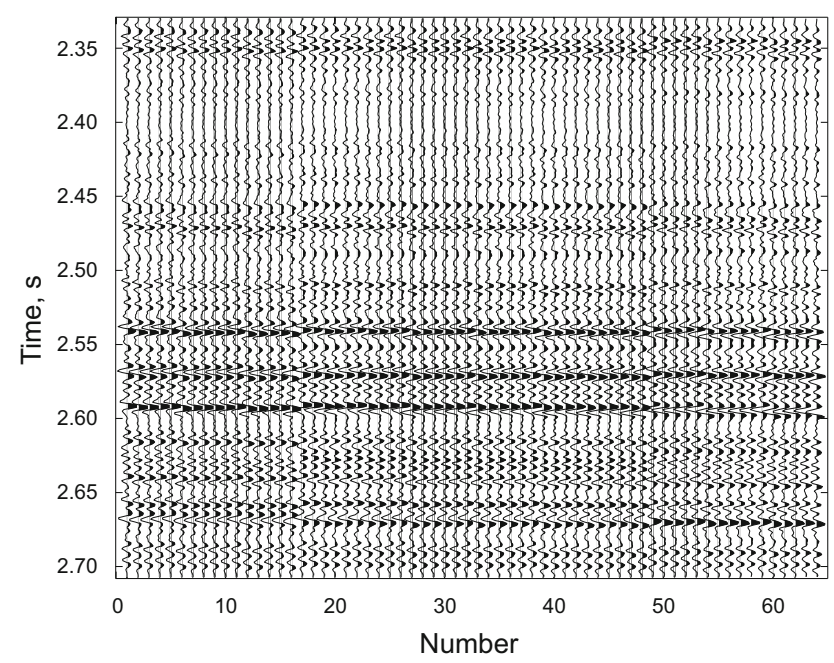

Fig. 9 Seismic record generated by the convolution of wavelets in Fig. 3 and reflectivity series in Fig. 6

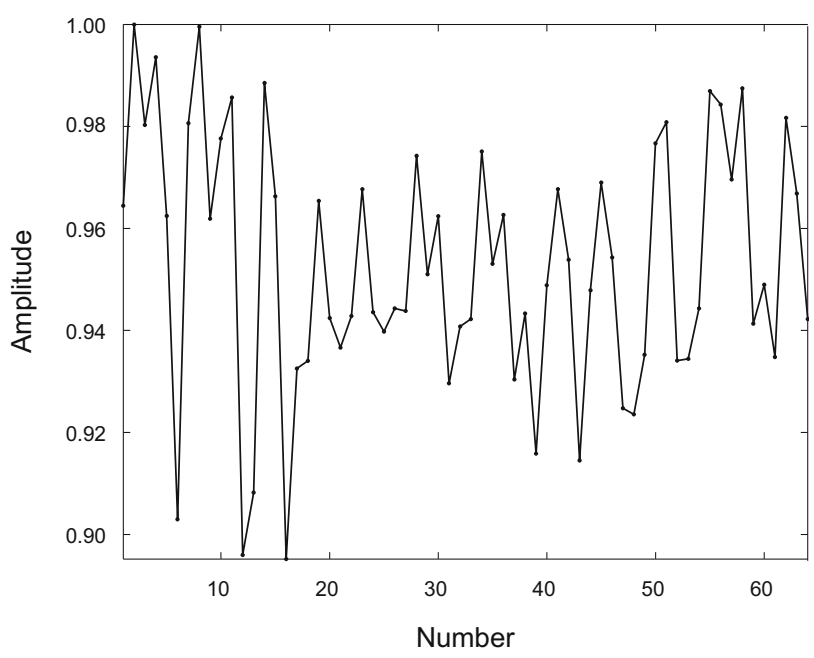

(a)

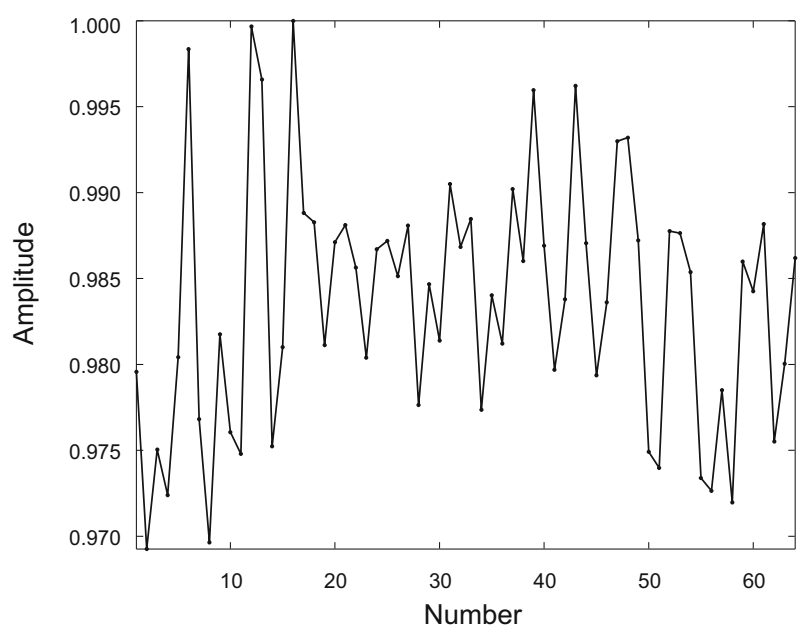

(b) 


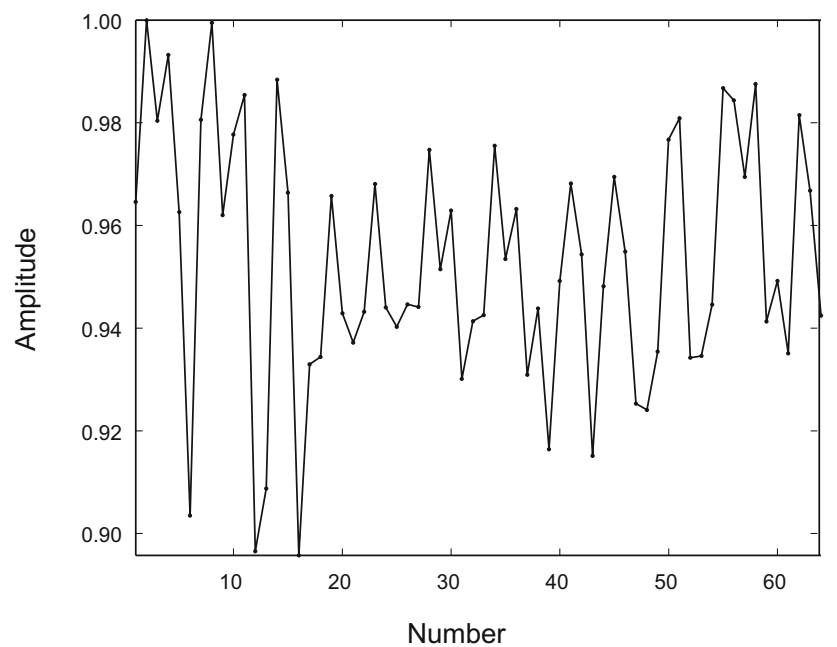

(c)

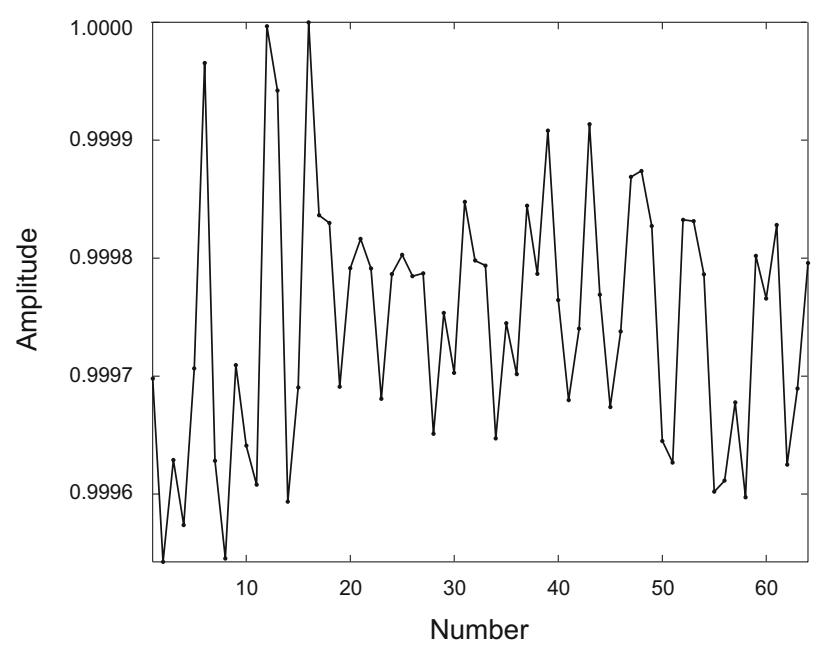

(d)

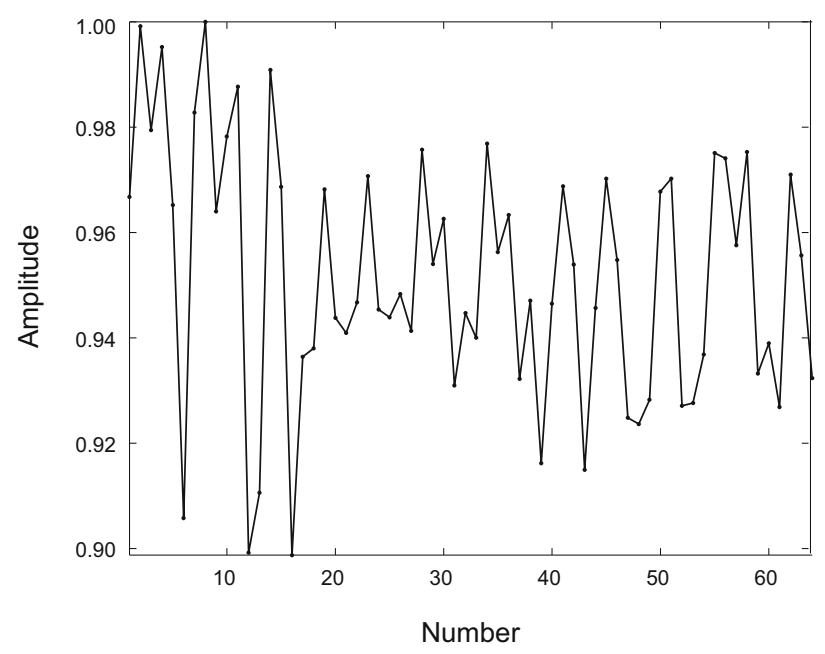

(e)

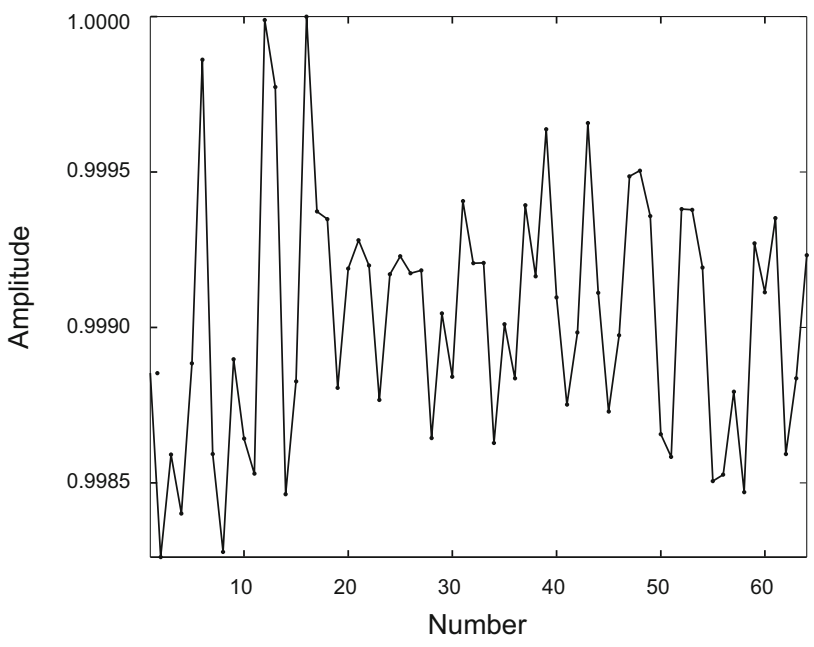

(f)

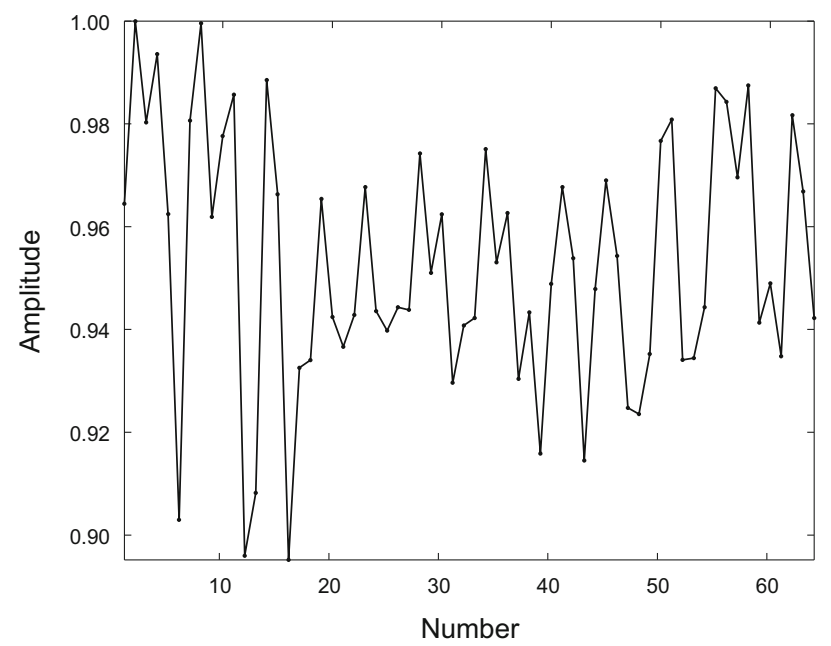

(g)

Fig. 10 The corresponding criteria function of every seismic trace in Fig. 9. (a) kurtosis criterion, (b) parsimony criterion, (c) Lu's improved kurtosis criterion, (d) Cauchy criterion, (e) exponential transform criterion, (f) modified Cauchy criterion, and (g) Sech criterion

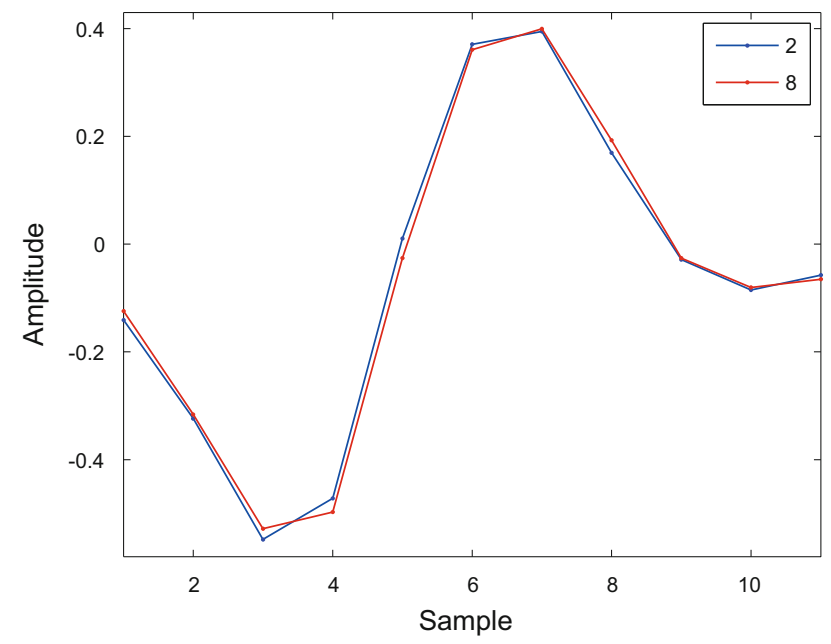

Fig. 11 The $2^{\text {nd }}$ and $8^{\text {th }}$ wavelet in Fig. 3 
wavelet, and all will be affected by the sparse feature of the reflectivity series. When the reflectivity series are sparse enough, as described in part four, the wavelet can be thought of as the seismic record, and the reflectivity series is a pulse, which is the most sparse reflectivity series. In this situation, for constant-phase and non-constant-phase wavelet, phase spectrum estimation based on criterion function can all obtain the true wavelet phase spectrum. When reflectivity series is non-sparse, even ignoring the impacts of other factors such as noise, as described in part five, the estimated phase spectra of constant-phase wavelets by criteria function all deviate from the true value of $28.8^{\circ}$, and those of non-constant-phase wavelet are also the same and deviate from the true value. Therefore, when applying the method to real seismic data, we need to make accurate judgments on the sparse feature of the reflectivity series, otherwise the estimated wavelet phase spectrum will likely deviate seriously from the true value.

\section{Seismic wavelet phase spectrum estimation based on criterion functions and multi traces}

When reflectivity series is not sparse enough, in order to improve the effect of seismic wavelet phase spectrum estimation based on criterion function, we apply the method to the multi-trace seismogram.

\subsection{Constant-phase wavelet}

Fig. 12 is the synthetic seismogram of a zero-phase wavelet and reflectivity series in Fig. 6. Considering that reflection coefficients in real strata have both consistency and tiny changes in horizontal direction, we introduce random disturbance to the original reflectivity series in every trace.

For a constant-phase wavelet, we make a constant-phase rotation on every trace, and calculate the criterion function, then obtain the criterion function of the whole seismogram by summing and averaging the criterion function of every trace. Considering that the kurtosis criterion, Lu's improved kurtosis criterion, exponential transform criterion, and Sech criterion have the similar characteristics, and the parsimony criterion, Cauchy criterion, and the modified Cauchy criterion have the similar characteristics, we show only representatives of the kurtosis criterion and the parsimony criterion (Fig. 13). We can see that the estimated phase spectrum of the constantphase wavelet is $14.4^{\circ}$. Compared with the result $\left(28.8^{\circ}\right)$ of a single trace, the accuracy is remarkably improved. Fig. 14 is comparison of estimated wavelet and true wavelet, and they are close to each other.

\subsection{Non-constant-phase wavelet}

Similarly to Fig. 12, we introduce random disturbance to reflectivity in Fig. 6 , and convolute them with the $2^{\text {nd }}$ mixed-phase wavelet in Fig. 3, thereby obtain the multi-trace seismogram (Fig. 15). We can obtain a series of wavelets that have different phase spectra by root transform, and eliminate the wavelet phase spectrum from the seismic trace. Then we calculate the criterion function of the single trace, sum and average them. Finally we obtain the criterion function of the whole seismogram. Here we also show only the kurtosis

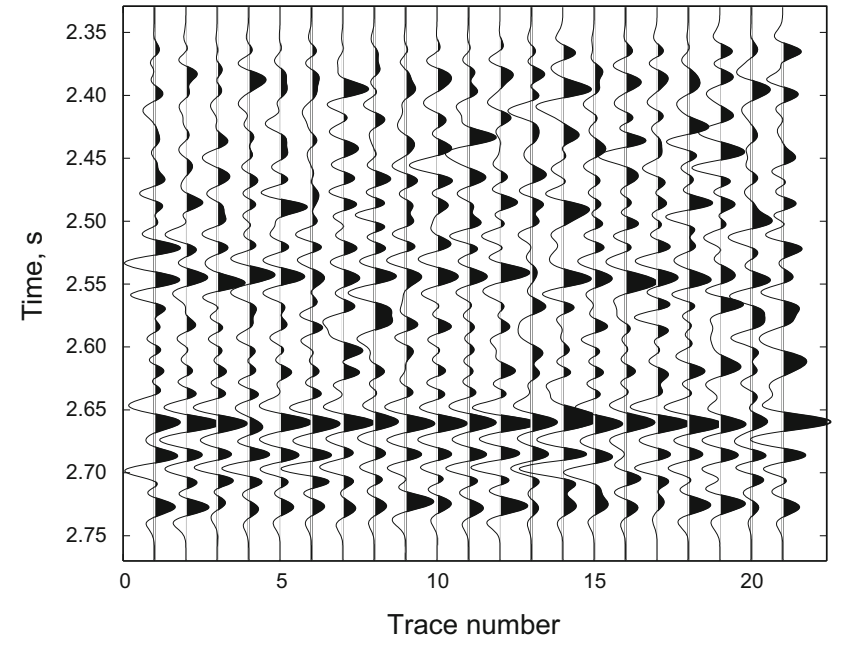

Fig. 12 Synthetic seismogram by using the zero-phase wavelet in Fig. 1

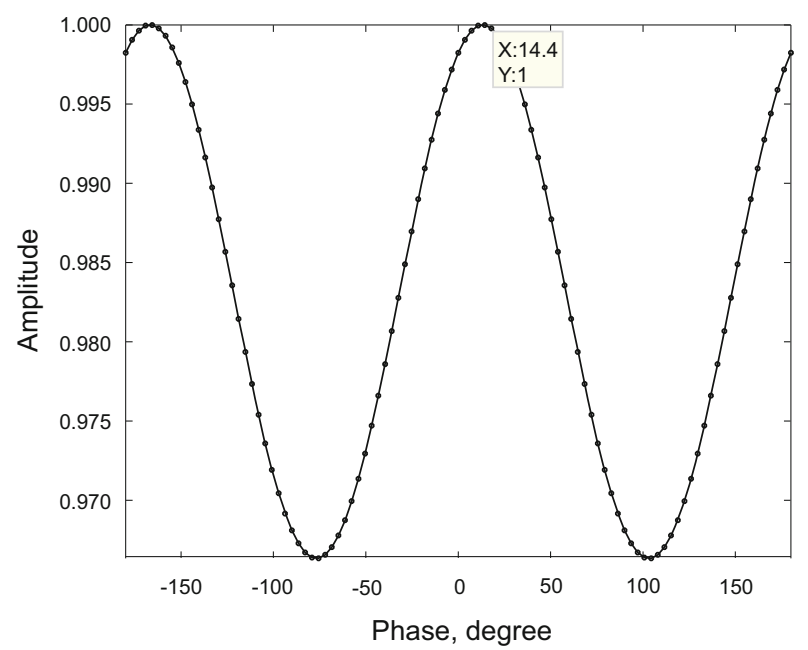

(a)

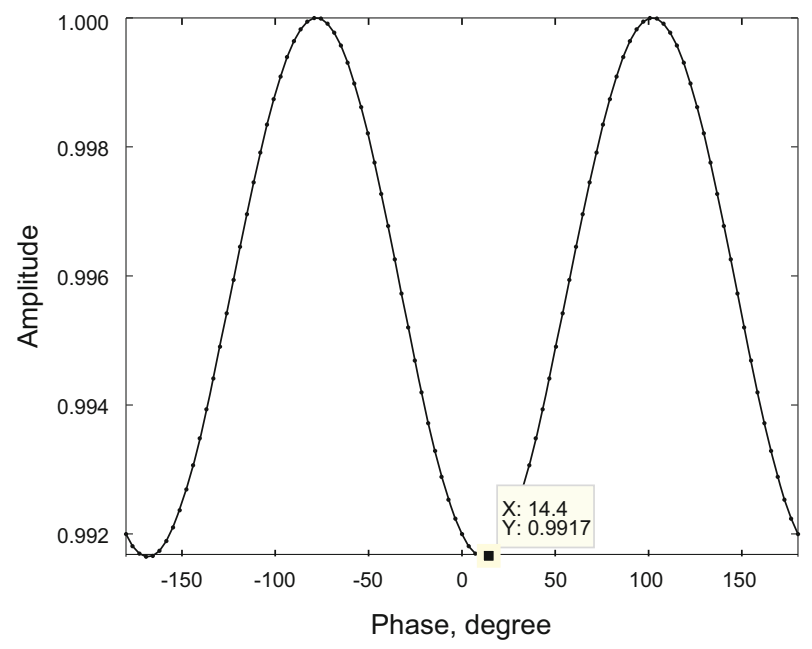

(b)

Fig. 13 Criteria function of the seismic wavelet phase spectrum estimation based on constant-phase rotation. (a) kurtosis criterion, and (b) parsimony criterion 


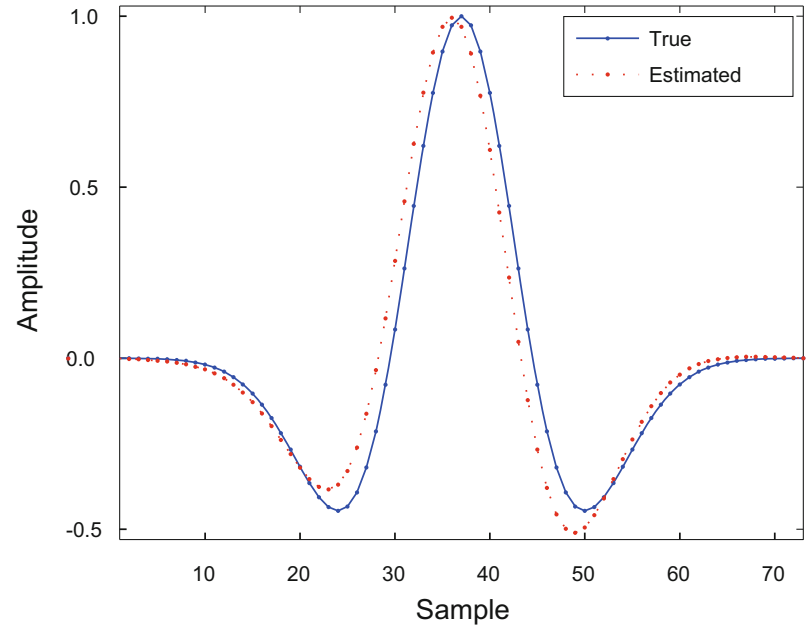

Fig. 14 Estimated wavelet and true wavelet

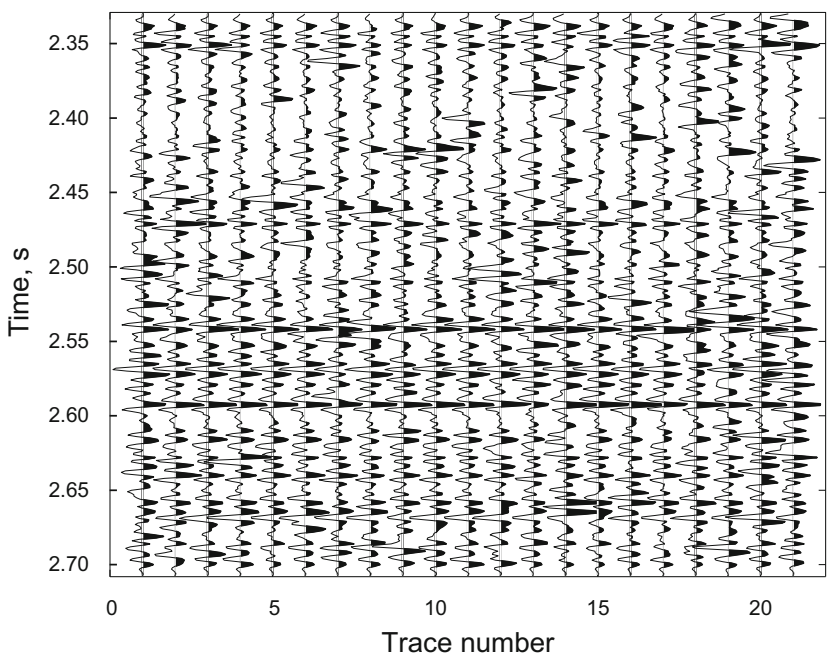

Fig. 15 Synthetic seismogram by using the $2^{\text {nd }}$ mixed-phase wavelet in Fig. 3

criterion and the parsimony criterion (Fig. 16). We can see that the kurtosis criterion of the $2^{\text {nd }}$ wavelet gives a maximum, and the parsimony criterion shows a minimum. We pick up this wavelet, and find that it is completely consistent with the true wavelet (Fig. 17). The effect of seismic wavelet phase spectrum estimation has been significantly improved.

\section{Conclusions}

Constant-phase rotation and root transform are two kinds of wavelet transform in seismic wavelet phase spectrum estimation based on criterion functions, which needs us to choose according to the characteristics of the data in real seismic data processing and interpretation.

The kurtosis criterion, Lu's improved kurtosis criterion, the parsimony criterion, exponential transform criterion, Sech criterion, Cauchy criterion, and the modified Cauchy criterion have a similar effect in phase spectrum estimation of seismic wavelet, and all will be affected by the sparse feature of the reflectivity series. When the reflectivity series is sparse enough, the results of phase spectrum estimation

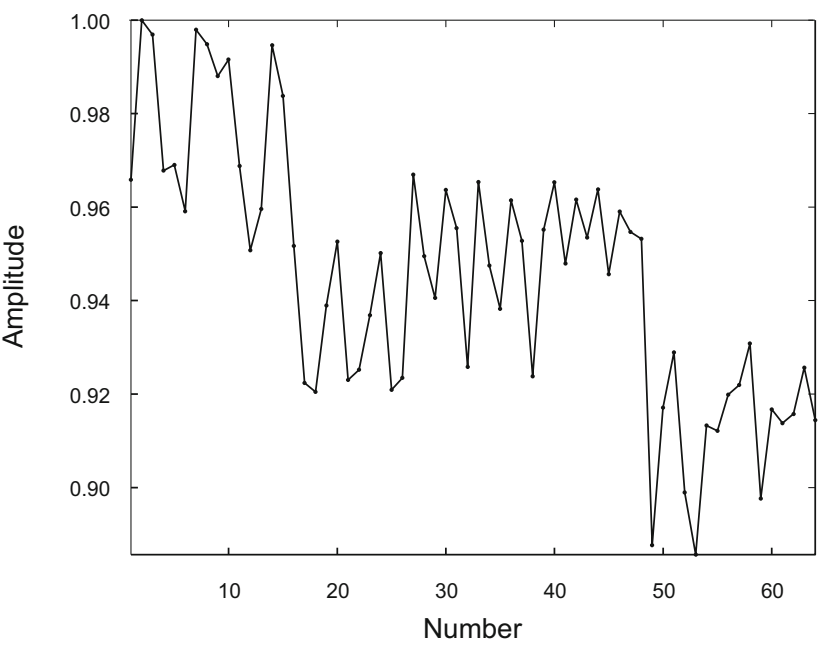

(a)

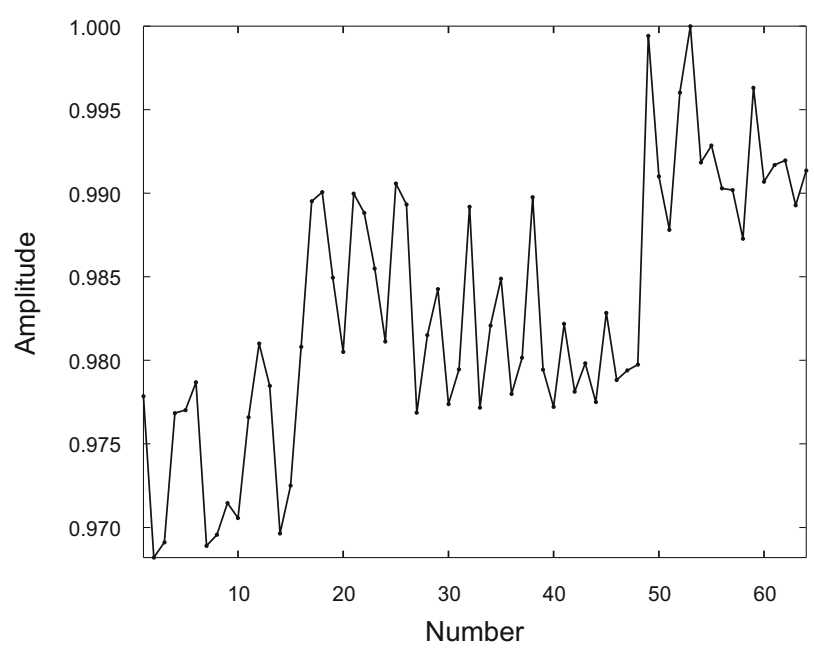

(b)

Fig. 16 Criteria function of seismic wavelet phase spectrum estimation based on root transform. (a) kurtosis criterion, and (b) parsimony criterion

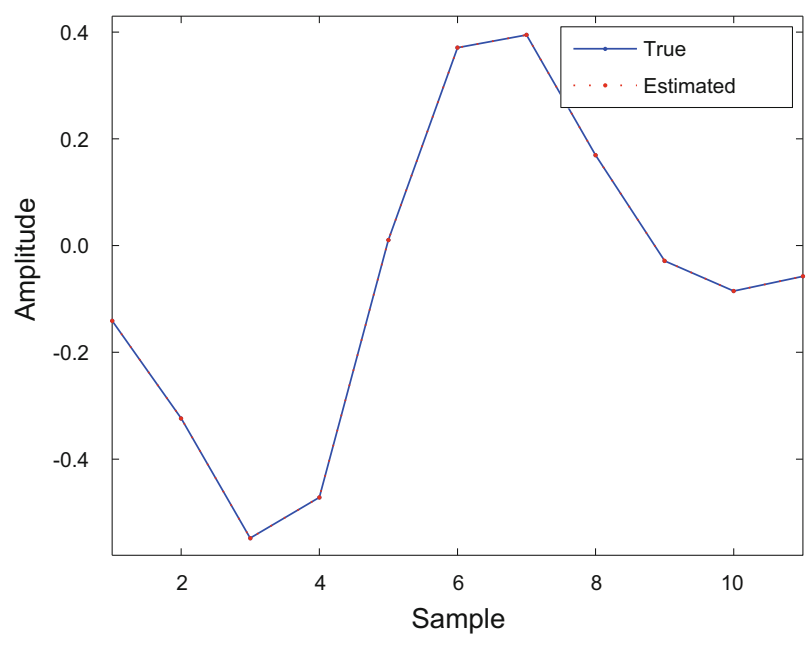

Fig. 17 Estimated wavelet and true wavelet

of the seismic wavelet are completely consistent with the true value. When the reflectivity series does not satisfy the sparse feature, even ignoring the impacts of other factors, 
the estimated wavelet phase spectrum by criterion function is still very different from the true value. Therefore, we need to make accurate judgments on the sparse feature of the reflectivity series in real seismic data.

In order to eliminate the influence of non-sparse reflectivity series in a single trace, we apply the method to a multi-trace seismogram, improving the accuracy of seismic wavelet phase spectrum estimation.

\section{Acknowledgements}

This research is supported by the Major Basic Research Development Program of China (973 Project No. 2007CB209608). The authors gratefully acknowledge their financial support.

\section{References}

Arons A B and Yennie D R. Phase distortion of acoustic pulses obliquely reflected from a medium of higher sound velocity. Journal of the Acoustical Society of America. 1950. 22(2): 231-327

Claerbout J F. Parsimonious deconvolution. Stanford Exploration Project. 1977. 13: 1-9

Edgar J A and van der Baan M. How reliable is statistical wavelet estimation? Geophysics. 2011. 76(4): V59-V68

Leinbach J. Wiener spiking deconvolution and minimum-phase wavelets: A tutorial. The Leading Edge. 1995. 14(3): 189-192

Levy S and Oldenburg D W. Automatic phase correction of commonmidpoint stacked data. Geophysics. 1987. 52(1): 51-59

Longbottom J, Walden A T and White R E. Principles and application of maximum kurtosis phase estimation. Geophysical Prospecting. 1988. 36(2): $115-138$

Lu W K. Frequency recovery of band-limited seismic data based on sparse spike train deconvolution and lateral coherence constraint. CPS/SEG Beijing 2009 International Geophysical Conference \& Exposition. 2009. ID: 1221

Lu W K. Non-minimum-phase wavelet estimation using second- and third-order moments. Geophysical Prospecting. 2005. 53(1): 149-158

Lu W K and Liu D Q. Frequency recovery of band-limited seismic data based on sparse spike train deconvolution. SEG/San Antonio 2007 Annual Meeting. 2007. 1977-1981

Lu W K, Zhang Y S, Zhang S W, et al. Blind wavelet estimation using a zero-lag slice of the fourth-order statistics. Journal of Geophysics and Engineering. 2007. 4(1): 24-30

Ooe M and Ulrych T J. Minimum entropy deconvolution with an exponential transformation. Geophysical Prospecting. 1979. 27(2): 458-473

Robinson E A. Predictive decomposition of time series with application to seismic exploration. Geophysics. 1967. 32(3): 418-484

Rosa A L R and Ulrych T J. Processing via spectral modeling. Geophysics. 1991. 56(8): 1244-1251

Sacchi M D. Statistical and Transform Methods in Geophysical Signal Processing. Canada: Department of Physics, University of Alberta. 2002

Sacchi M D, Velis D R and Cominguez A H. Minimum entropy deconvolution with frequency-domain constraints. Geophysics. 1994. 59(6): 938-945

Van der Baan M. Time-varying wavelet estimation and deconvolution by kurtosis maximization. Geophysics. 2008. 73(2): V11-V18

Van der Baan M and Fomel S. Nonstationary phase estimation using regularized local kurtosis maximization. Geophysics. 2009. 74(6): A75-A 80

White R E. Maximum kurtosis phase correction. Geophysical Journal International. 1988. 95(2): 371-389

Wiggins R A. Minimum entropy deconvolution. Geoexploration. 1978. 16(1-2): 21-35

Yu Y C, Wang S X, Yuan S Y, et al. Phase estimation in bispectral domain based on conformal mapping and applications in seismic wavelet estimation. Applied Geophysics. 2011. 8(1): 36-47

Yuan S Y and Wang S X. Influence of inaccurate wavelet phase estimation on seismic inversion. Applied Geophysics. 2011. 8(1): 48-59

Yuan S Y, Wang S X and Tian N. Swarm intelligence optimization and its application in geophysical data inversion. Applied Geophysics. 2009. 6(2): 166-174

Zhang F C, Liu J, Yin X Y, et al. Modified Cauchy-constrained seismic blind deconvolution. Oil Geophysical Prospecting. 2008. 43(4): 391396 (in Chinese)

(Edited by Hao Jie) 